\title{
La enseñanza de endemismos mexicanos a través de video documental e infografía, un ejemplo de Aprendizaje Basado en Proyectos
}

\section{Teaching Mexican endemisms through video documentaries and infographics, an example of Project Based Learning}

\author{
DOI: $10.46932 / \mathrm{sfjdv2n4-036}$
}

Received in: May 1st, 2021

Accepted in: Jun 30th, 2021

\section{García-Cruz Karla Verónica \\ $\mathrm{PhD}$ en Ciencias}

Escuela Nacional Preparatoria No.8 “Miguel E. Schulz”, Universidad Nacional Autónoma de México Av. Lomas De Plateros S/N, Merced Gómez, Álvaro Obregón, 01600 Ciudad de México, CDMX

E-mail: veronica.garcia@enp.unam.mx

\section{Cruz-Sánchez David}

Master en Ciencias Biológicas

Escuela Nacional Preparatoria No.8 "Miguel E. Schulz", Universidad Nacional Autónoma de México Av. Lomas De Plateros S/N, Merced Gómez, Álvaro Obregón, 01600 Ciudad de México, CDMX

E-mail: david.cruz@enp.unam.mx

\section{RESUMEN}

En este proyecto se describe la experiencia durante la aplicación de 2 Tecnologías de la Información: el video documental y la infografía, como medios para adquirir aprendizajes significativos sobre el tema: Endemismos mexicanos: causas, distribución e importancia. Este tema pertenece al programa de Biología IV del bachillerato de la UNAM. Las TIC fueron desarrolladas por los alumnos de quinto año de bachillerato de dos grupos diferentes. La finalidad de este trabajo es que los alumnos emplearán diversas herramientas colaborativas como Google Docs para realizar un proyecto cuyo producto fuera un video documental y una infografía, sobre el tema antes señalado. Los resultados de ambos trabajos aportan evidencias de aprendizaje colaborativo, siendo Google Docs una herramienta útil para el trabajo colaborativo asíncrono. Finalmente, este trabajo aporta dos opciones de productos que el docente puede emplear para desarrollar un tema. Siendo el video y la infografía un producto más significativo para construir el pensamiento crítico, valores y cambios de actitudes.

Palabras clave: Infografía, video documental, Biología, TIC-TAC, ABP, TIC-TAC.

\begin{abstract}
This project describes the experience during the application of two information technologies: video documentaries and infographics, as a means to acquire significant learning on the topic: Mexican endemisms: causes, distribution and importance. This topic belongs to the Biology IV program of the UNAM high school. The ICT were developed by fifth year high school students from two different groups. The purpose of this work is that students will use various collaborative tools such as Google Docs to carry out a project whose product was a documentary video and an infographic on the topic mentioned above. The results of both projects provide evidence of collaborative learning, being Google Docs a useful tool for asynchronous collaborative work. Finally, this work provides two options of products that the teacher can use to develop a topic. Video and infographics being a more significant product to build critical thinking, values and attitude changes.
\end{abstract}


Key words: Infographics, documentary video, Biology, ICT-CT, PBL, ICT-CT.

\section{INTRODUCCIÓN}

El programa de Biología IV de la Escuela Nacional Preparatoria (ENP) de la UNAM contempla entre sus ejes transversales, la construcción del conocimiento empleando las Tecnologías de la Información y Comunicación (TIC) (DGENP. 2013). Estas herramientas no sólo facilitan la información, también pueden ser adecuadas para favorecer el aprendizaje convirtiéndose en Tecnologías para el Aprendizaje y el Conocimiento (TAC). Estas tecnologías aportan al docente nuevas estrategias didácticas para abordar diversos contenidos (Ortiz Marín, et al., 2021).

Del mismo modo, plantea el trabajo colaborativo a través del Aprendizaje Basado en Proyectos o ABP. Este tipo de estrategia se enfoca en un problema que hay que solucionar, o una situación que hay por analizar, en base a un plan de acción en donde los estudiantes identifican el ¿qué?, ¿para qué? ¿cómo? ¿con quién? ¿para cuándo? ¿dónde? ¿con cuánto? ¿con qué?, riesgos a enfrentar, medidas alternativas para asegurar el éxito, resultados esperados, entre otros (Alvarado, 2017). Esta estrategia está centrada básicamente en el alumno, en donde desarrolla un conjunto de actividades interdisciplinarias de mediano y largo plazo, bajo su propia responsabilidad. Pueden identificarse ciertas etapas en el proceso, como son, inicio: En donde se define el tema o tópico, discusión con toda la clase, objetivos, metas a cumplir y métodos de evaluación del proyecto. La siguiente etapa es la referente a la planeación del trabajo, la división de proyecto en sus componentes y asignar las responsabilidades entre los miembros del equipo, así como los mecanismos de retroalimentación por parte del profesor. La siguiente etapa consta del desarrollo de proyecto, aquí el profesor juega el papel de facilitador para que los estudiantes completen las tareas, en esta etapa los miembros del equipo toman parte en el aprendizaje colaborativo y en la solución cooperativa de los problemas. Asimismo se llega al resultado final con un producto, presentación o una interpretación dirigida a una audiencia específica. En la última etapa, la de conclusión desde la perspectiva de los estudiantes, se hace la revisión final por parte de los alumnos y el profesor con la finalidad de dar la presentación final. En la evaluación final se presenta el trabajo terminado en forma acordada. Por lo general, toda la clase participa ofreciendo una retroalimentación constructiva. El proceso termina con una reflexión por parte de estudiantes en donde analizan sus productos; mientras que el profesor revisa el funcionamiento de la secuencia didáctica y las áreas de oportunidad para la siguiente ocasión (Alvarado, 2017).

Por otra parte, el contenido conceptual "Endemismos mexicanos: causas distribución e importancia", puede ser abordado mediante un ABP. En donde los alumnos empleen TIC como Google Drive o plataformas académicas para la construcción de proyectos TAC como documentales o infografías. 
Con el trabajo colaborativo, los integrantes de un equipo pueden exponer conocimientos, materiales e ideas con la finalidad de compartirlos, para construir un conocimiento común que se pueda utilizar globalmente (Domingo-Coscollola, 2014) Para ello, los alumnos se tienen que reunir en sesiones extraclase, acordar horarios y organizar su carga de trabajo, por lo que, en muchas ocasiones, resulta complicada la organización presencial. En la actualidad, el trabajo colaborativo tiene la posibilidad de llevarse a cabo en línea, lo cual permite al equipo comunicarse en tiempo real, a pesar de la distancia y lugar. Por esta razón, aprender y desarrollar habilidades de trabajo colaborativo en línea con el uso de herramientas de Google es indispensable y de gran utilidad para el trabajo.

\section{DESARROLLO}

"Endemismos mexicanos: causas, distribución e importancia" es uno de los nuevos contenidos presentes en el programa de Biología IV. En este tema se revisan los conceptos de endemismos, las causas físico-químicas que los originaron y la importancia ecológica de una especie con distribución restringida. Además, requiere que los alumnos valoren la pérdida de una especie, así como el papel que juegan en su conservación, esto con el objetivo de que los alumnos adquieran un cambio en su actitud sobre la pérdida de una especie endémica.

Este trabajo se realizó con alumnos del quinto año de la ENP de la UNAM en el ciclo escolar 2017-2018. En la primera actividad, los alumnos formaron equipos de 5 a 6 integrantes. Posteriormente, investigaron en fuentes confiables la información referente a una especie endémica mexicana de su preferencia, la información fue concentrada en un documento colaborativo de Google Docs. La información debía contener:
a) nombre común y científico de la especie.
b) clasificación taxonómica.
c) hábitat.
d) alimentación.
e) nicho ecológico.
f) problemas que lo amenazan.
g) conclusiones.
h) referencias.

En la actividad número 2, los alumnos elaboraron un guión para realizar una entrevista a un investigador, tesista o especialista en la especie endémica. A los alumnos se les sugirió entrevistar al investigador presencialmente o por videoconferencia. Entre las características del guión se encontraba añadir los tiempos, preguntas y participación de los integrantes. Además debían incluir evidencia de la 
colaboración. Esta evidencia se basó en el empleo del chat y/o comentarios, las cuales son herramientas del Google Docs. A continuación, se muestran capturas de pantalla de dichas evidencias. Como se muestra en la foto no solo les sirvió a los alumnos como medio de comunicación entre pares, además al docente como herramienta para retroalimentar el trabajo durante su realización.

Figura 1. Guión de uno de los videos con evidencia de trabajo colaborativo en línea.

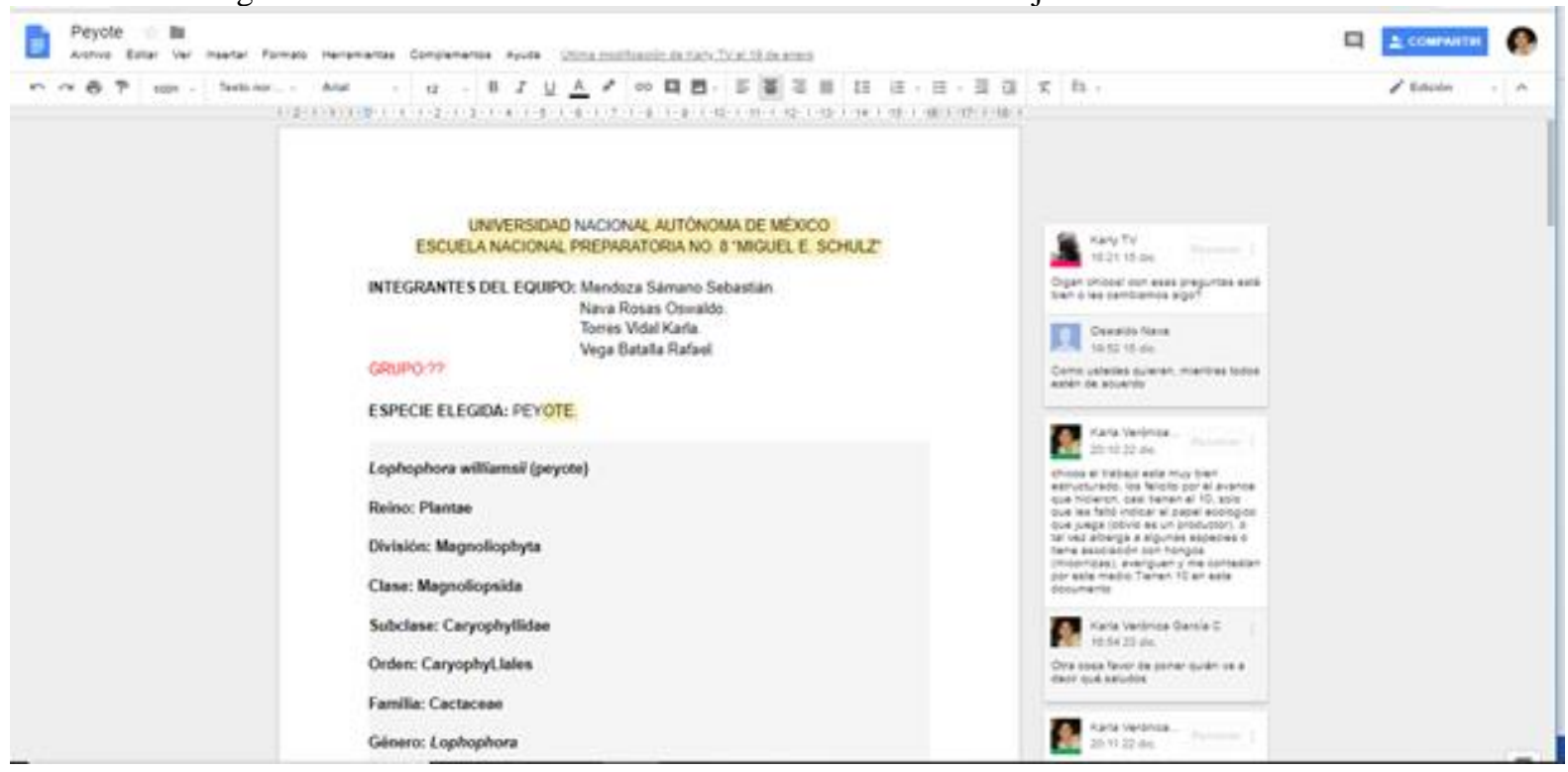

Actividad número 3. Con la finalidad de desarrollar destrezas en la elaboración de video como edición, producción, estética, síntesis de la información, así como manejo de software especializado; se elaboró un video en formato de documental. Este cumple con el propósito de transmitir la información de los expertos, así como valores. El video tiene las siguientes características:

a) Cumplimiento con las especificaciones descritas en el guión.

b) Estética.

c) Producción y edición.

d) Difusión en YouTube.

Entre los videos obtenidos, se observó que los alumnos trabajaron con distintas aplicaciones como: Filmora, Movie maker, Filmmaker, Promovavi, Powtoon, entre otros.

Las siguientes fotografías muestran las evidencias de los videos elaborados por los alumnos y su difusión en YouTube. 
Figura 3. Ejemplos de los videos obtenidos y su difusión en YouTube

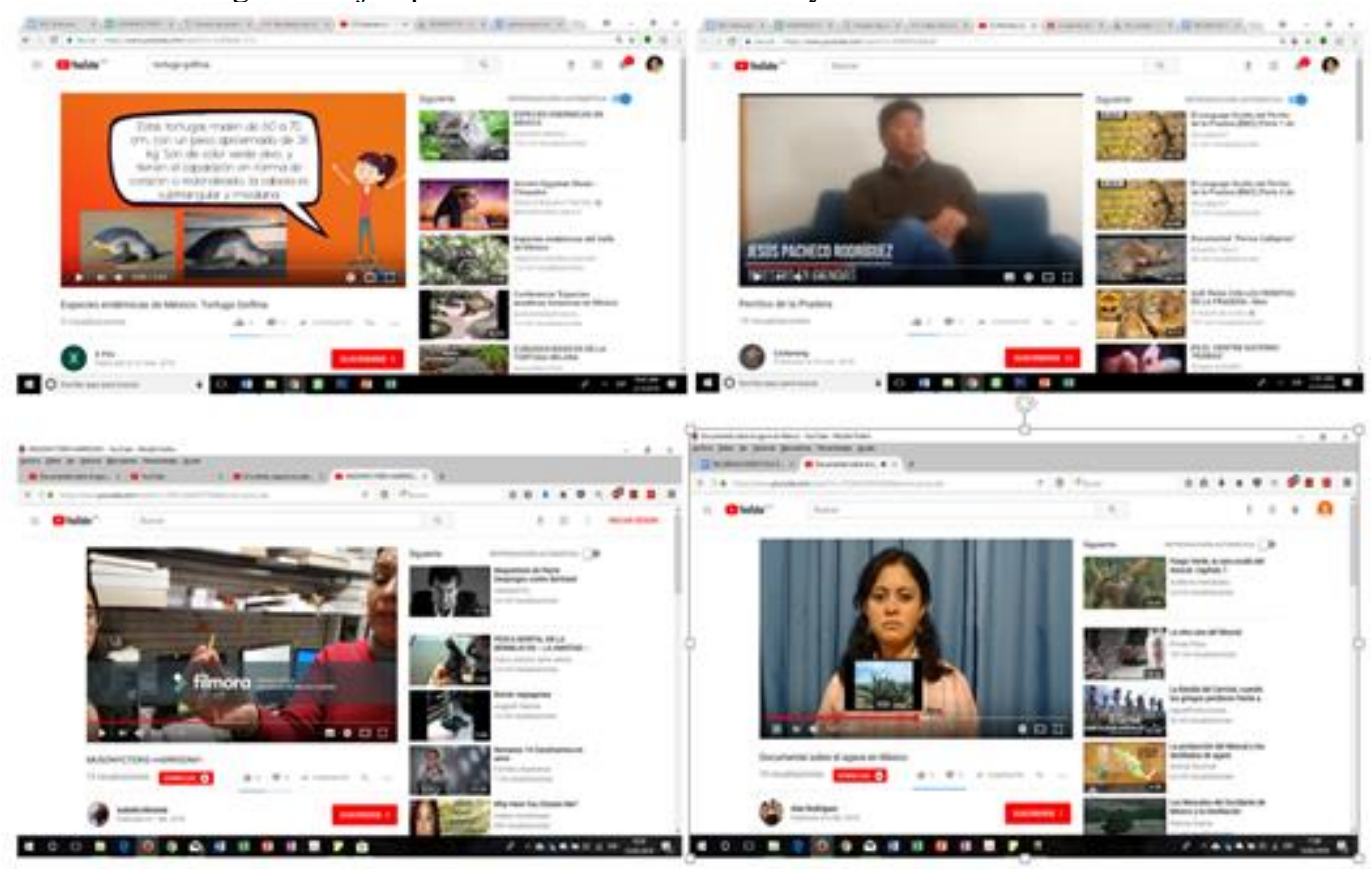

Una vez que el video fue retroalimentado y evaluado, los alumnos lo presentaron ante el grupo. Un ejemplo representativo de este trabajo se puede encontrar en:https://youtu.be/_5VF09XTRus . Dicho trabajo se presentó en la feria de alumnos, encuentro de profesores del programa Dominó-TIC de la ENP y en el EDUCATIC de DGTIC de la UNAM.

Figura 4. Exposición de los trabajos frente a grupo.

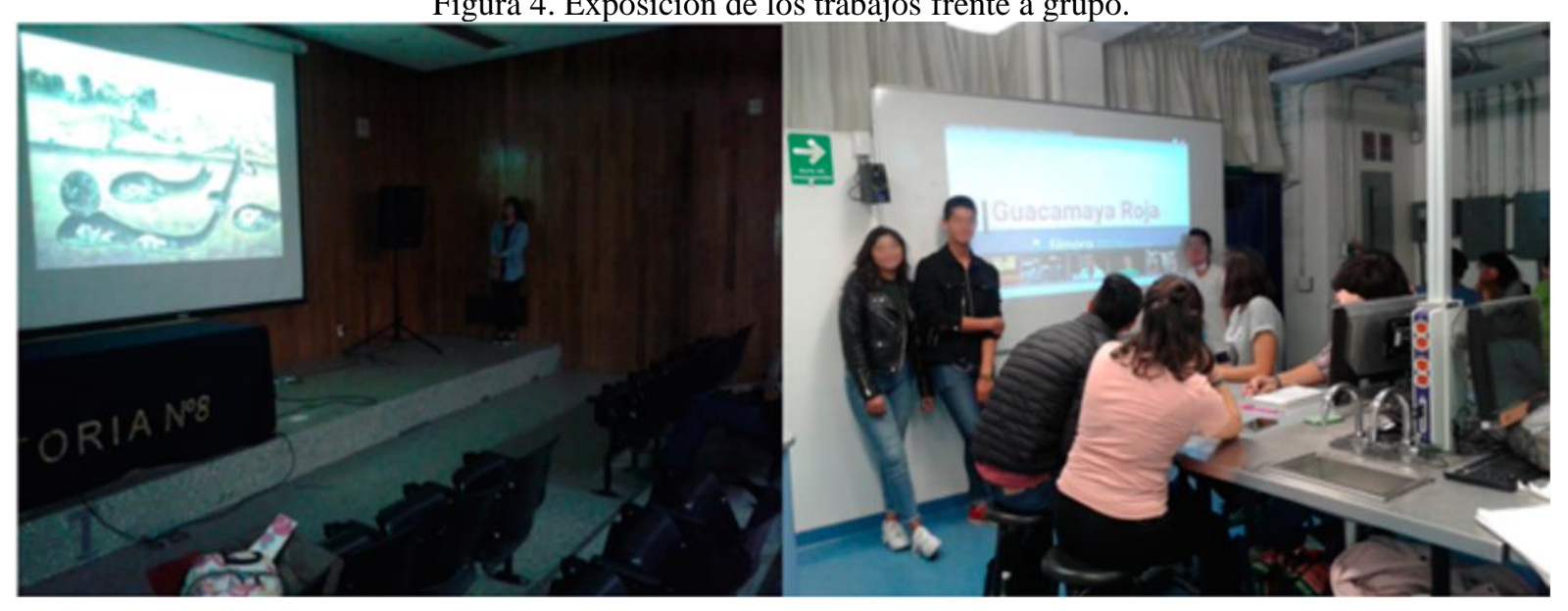

Otra herramienta en donde los alumnos desarrollan habilidades como organización, síntesis de la información, manejo de imágenes y participación colaborativa, se encuentra en las infografías.

Para ello se pidió a alumnos de otro grupo que elaborarán infografías de la especie endémica empleando la aplicación de Piktochart. 


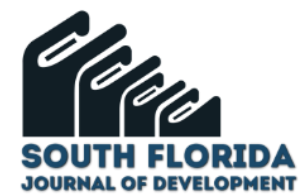

Los alumnos elaboraron está TAC con las especificaciones descritas para la investigación inicial, empleando las fuentes bibliográficas confiables que ellos desearan. Posteriormente, las infografías fueron publicadas en el aula virtual, en donde recibieron retroalimentación por parte de los profesores y de sus compañeros.

A continuación, se describen algunas evidencias de las infografías presentadas por los alumnos y la retroalimentación presentada por los profesores (Figura 5).

Figura 5. Infografía presentada y evidencia de retroalimentación por parte de la profesora.

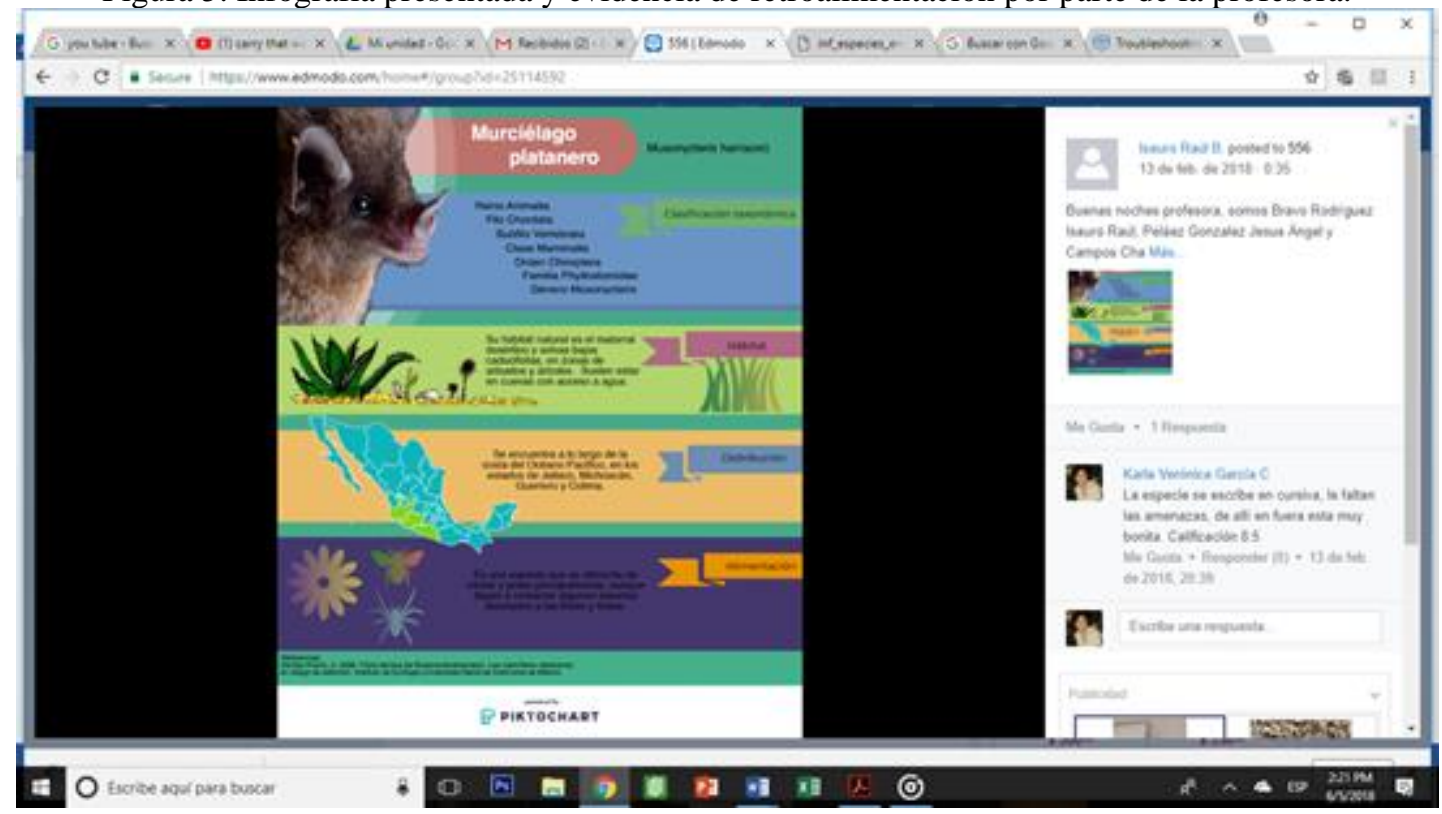

Figura 6. Infografías representan algunos de los trabajos recibidos.

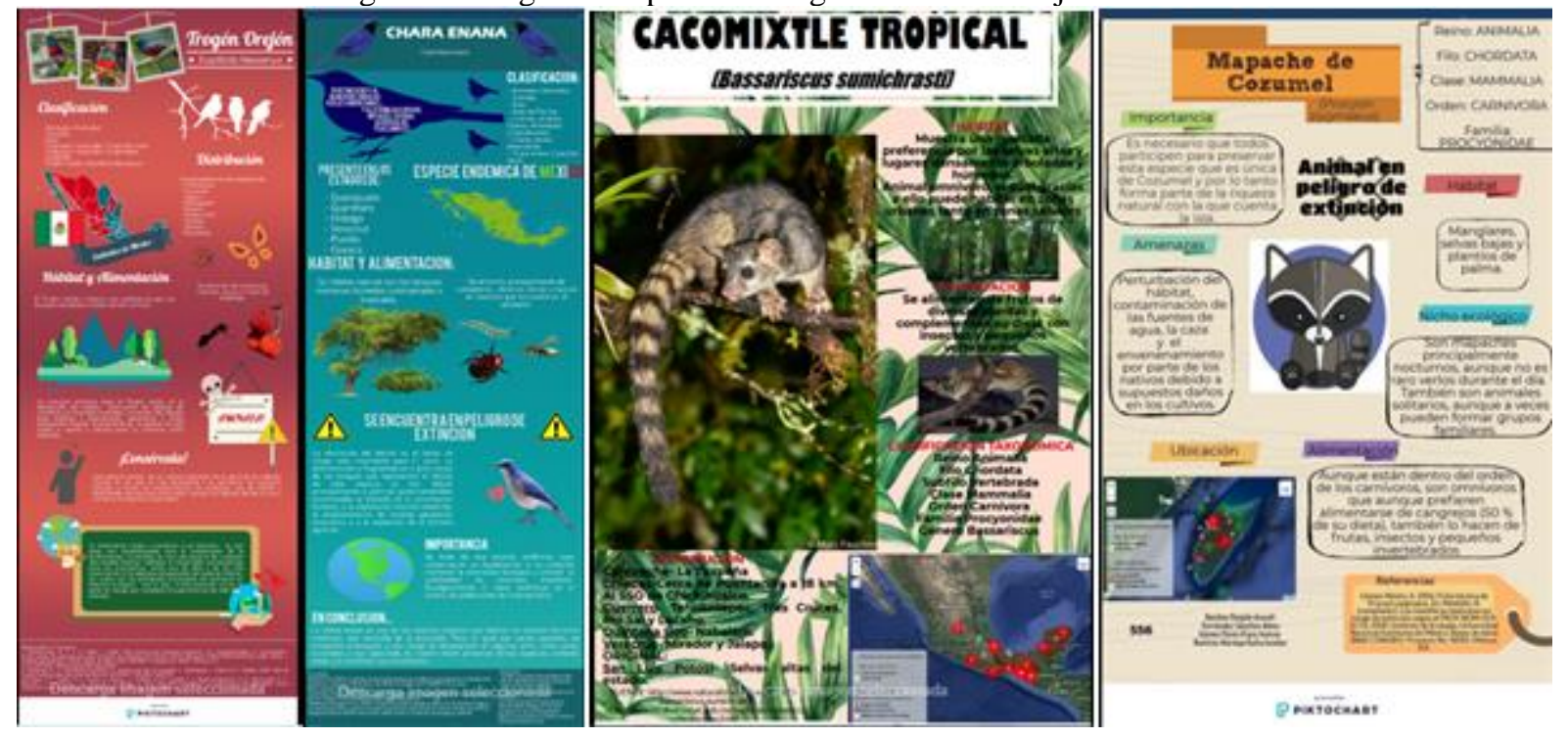




\section{CONCLUSIONES Y APORTES DEL TRABAJO}

Este tipo de proyectos, en donde los alumnos construyen su conocimiento utilizando TIC para elaborar TAC, no solo repercute en el área del conocimiento, también en la actitud con respecto a los problemas actuales. Esta última área resulta difícil de estimar debido a que no basta detectarla por medio de evaluaciones habituales. Sin embargo, al cuestionar a los alumnos sobre las impresiones que dejó en ellos su proyecto, todos comentaron sobre la relevancia de conservar a las especies, para mantener el equilibrio ecológico. Además, refirieron que la difusión de sus trabajos en plataformas es un primer paso para hacer algo por ellas. Como se aprecia en el video representativo, los alumnos cuestionaron al especialista sobre las formas para ayudar a su especie estudiada, esto fue iniciativa propia (se sugiere ver video https://youtu.be/_5VF09XTRus?t=342 minuto 5, 41.

En cuanto a la estrategia didáctica empleada, es un ejemplo de Aprendizaje Basado en Proyectos (ABP), ya que cumple con las características de: desarrollo de habilidades y competencias, como se observa durante la elaboración de los videos; promueve el aprendizaje significativo y se desarrolla en grupos de trabajo, en la búsqueda de resolver un problema retador para el estudiante (Alvarado, 2017) . Además la desaparición de especies endémicas resulta ser un problema actual, conocido por los estudiantes, por lo que no representa un problema ajeno. Además este ABP modifica la concepción tradicional de proceso enseñanza-aprendizaje, otra característica de un ABP, en donde el profesor es el centro de enseñanza. En el presente proyecto, este proceso se centra en el alumno, tal como se ha observado en recientes trabajos en donde los participantes del ABP son sujetos activos y reguladores de su proceso enseñanza-aprendizaje (García-Cruz y Cruz-Sánchez. 2021).

Por otra parte, el proyecto propone un video en formato documental y una infografía, siendo productos alcanzables a corto y mediano plazo para los estudiantes. Esta decisión dependerá de los alcances del profesor para el andamiaje y evaluación, ya que una de las características del ABP es que suelen tener una carga de trabajo mayor para el profesor Aunque en gran medida eso se resuelve con el uso de las TIC-TAC. Debido a que el profesor puede revisar y compartir los avances de los alumnos por medio de los documentos compartidos de Google Docs, cuyo beneficio para este último es la retroalimentación desde cualquier dispositivo, momento y lugar.

Entre las áreas de oportunidad identificadas para este trabajo se encuentran:

a) Retroalimentar una versión preliminar del video, para hacer apuntes principalmente en síntesis de la información presentada;

b) La elección de los entrevistados, ya que en algunos casos no fue la más adecuada;

c) Las infografías deben difundirse en periódicos murales, para que toda la comunidad conozca la importancia de conservar las especies endémicas. 
Por otra parte, la difusión de los trabajos en más foros académicos con otros alumnos, como coloquios que se realizan en diferentes planteles de la Escuela Nacional Preparatoria, hubiera enriquecido en buena forma la experiencia y motivación de los alumnos.

La difusión de estos videos por medio de Youtube tiene un alcance superior a la comunidad de la ENP, por lo que el contenido y su mensaje pueden llegar a más personas y transmitir valores sobre cuidado y conservación. 


\section{REFERENCIAS BIBLIOGRÁFICAS}

Alvarado, Clara. 2017. "Aprendizaje Basado en Proyectos". Proyecto Aula del Futuro. Espacios y Sistemas Interactivos para la Educación. Centro de Ciencias Aplicadas y Desarrollo Tecnológico UNAM. México.

DGENP. (2013). Avances del Proyecto de Modificación curricular. Recuperado 12 de junio de 2020 de http://proyectomc.dgenp.unam.mx/proyecto-de-modificacion/avances

Díaz-Barriga, F. Enseñanza Situada: Vínculo entre la escuela y la vida. (2006). Editorial Mc Graw Hill.

Domingo-Coscollola, M.; Sánchez-Valero, J. A.; Sancho-Gil, J. M. (2014). Investigar con y sobre los jóvenes colaborando y educando. Comunicar 42 (21): 157-164.

García-Cruz K. V. y Cruz-Sánchez D. (2021). Proyecto e-Book, una TAC para el aprendizaje centrado en el alumno. South Florida Journal of Development, Miami, v.2, n.2, p. 2607-2614. DOI: https://doi.org/10.46932/sfjdv2n2-116.

Google Docs. Español cómo utilizar Google documentos. https://www.youtube.com/watch?v=pUEwfZaumc8. Consultado el 22 de agosto de 2021.

Ortiz Marín P., Giménez Velázquez A. B., Rabal Alonso J. M. 2021. Percepción del profesorado acerca de la importancia del uso de las tic para favorecer la inclusión del alumnado. South Florida Journal of Development. Vol. 2 No. 2. DOI: https://doi.org/10.46932/sfjdv2n2-089

Programa Biología IV ENP. http://enp.unam.mx/assets/pdf/planesdeestudio/5to/1502_biologia_IV.pdf. Recuperado el 18 de agosto de 2021. 